\title{
A unifying framework for the SISO circle criterion and other quadratic stability criteria
}

\author{
Robert N. Shorten, Oliver Mason † Fiacre O’Cairbre ${ }^{\ddagger}$ and Paul Curran $\S$
}

\begin{abstract}
We present a result on the existence of a common quadratic Lyapunov function for a pair of linear time-invariant systems. We show that this result characterises, generalises, and provides new perspectives on several well-known stability results.
\end{abstract}

\section{Introduction}

In this paper we consider the problem of determining necessary and sufficient conditions for the existence of a common quadratic Lyapunov function (CQLF) for a pair of stable linear time-invariant (LTI) systems. This problem arises in many areas of systems theory; in particular, in the study of non-linear Lur'e type systems and in the study of switched linear systems. While the general algebraic problem is extremely difficult, necessary and sufficient conditions for various system classes have been obtained by reposing the problem in the form of a linear matrix inequality, or (by means of the positive real lemma) as a frequency-domain optimization. We present a new approach to solving this problem. By formulating the CQLF existence problem in a set-theoretic context, and by making simplifying assumptions, we obtain a simple eigenvalue condition for the existence of a CQLF for a pair of LTI systems. We show that well known stability criteria are characterised by this result. In particular, we obtain a new time-domain formulation of the circle criterion.

\footnotetext{
*Hamilton Institute, NUI Maynooth, Kildare, Ireland, robert.shorten@may.ie (joint first author)

${ }^{\dagger}$ Hamilton Institute, NUI Maynooth, Kildare, Ireland (joint first author)

${ }^{\ddagger}$ Mathematics Dept., NUI Maynooth, Kildare, Ireland

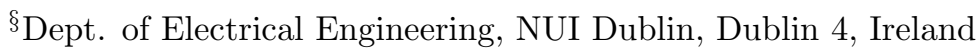




\section{Mathematical preliminaries and notation}

In this section we present preliminary results that are useful in deriving the main contribution of this paper. Throughout, the following notation is adopted: $\mathbb{R}$ and $\mathbb{C}$ denote the fields of real and complex numbers respectively; $\mathbb{R}^{n}$ denotes the $n$-dimensional real Euclidean space; $\mathbb{R}^{n \times n}$ denotes the space of $n \times n$ matrices with real entries; $x_{i}$ denotes the $i^{t h}$ component of the vector $x$ in $\mathbb{R}^{n} ; a_{i j}$ denotes the entry in the $(i, j)$ position of the matrix $A$ in $\mathbb{R}^{n \times n}$.

The main results of this paper are based upon Theorem 2.1. The concepts of weak quadratic Lyapunov functions, strong quadratic Lyapunov functions, and matrix pencils, are central to the statement of this theorem. Where appropriate, proofs of individual theorems and lemmas are given in the appendix.

(i) Strong and weak common quadratic Lyapunov functions : Consider the set of LTI systems

$$
\Sigma_{A_{i}}: \dot{x}=A_{i} x, i \in\{1,2, \ldots M\} .
$$

where $M$ is finite and the $A_{i}, i \in\{1,2, \ldots M\}$, are constant Hurwitz matrices in $\mathbb{R}^{n \times n}$ (i.e. the eigenvalues of $A_{i}$ lie in the open left half of the complex plane and hence the $\Sigma_{A_{i}}$ are stable LTI systems). Let the matrix $P=P^{T}>0, P \in \mathbb{R}^{n \times n}$, be a simultaneous solution to the Lyapunov equations

$$
A_{i}^{T} P+P A_{i}=-Q_{i}, i \in\{1,2, \ldots M\} .
$$

Then, $V(x)=x^{T} P x$ is a strong quadratic Lyapunov function for the LTI system $\Sigma_{A_{i}}$ if $Q_{i}>0$, and is said to be a strong CQLF for the set of LTI systems $\Sigma_{A_{i}}$, $i \in\{1, \ldots, M\}$, if $Q_{i}>0$ for all $i$. Similarly, $V(x)$ is a weak quadratic Lyapunov function for the LTI system $\Sigma_{A_{i}}$ if $Q_{i} \geq 0$, and is said to be a weak CQLF for the set of LTI systems $\Sigma_{A_{i}}, i \in\{1, \ldots, M\}$, if $Q_{i} \geq 0$ for all $i$.

(ii) The matrix pencil $\sigma_{\gamma[0, \infty)}\left[A_{1}, A_{2}\right]$ : The matrix pencil $\sigma_{\gamma[0, \infty)}\left[A_{1}, A_{2}\right]$, for $A_{1}, A_{2} \in$ $\overline{\mathbb{R}^{n \times n}}$, is the parameterised family of matrices $\sigma_{\gamma[0, \infty)}\left[A_{1}, A_{2}\right]=A_{1}+\gamma A_{2}, \gamma \in$ $[0, \infty)$. We say that the pencil is non-singular if $\sigma_{\gamma[0, \infty)}\left[A_{1}, A_{2}\right]$ is non-singular for all $\gamma \geq 0$. Otherwise the pencil is said to be singular. Further, a pencil is said to be Hurwitz if its eigenvalues are in the open left half of the complex plane for all $\gamma \geq 0$. It is important for much of what follows to note that when $A_{1}$ is non-singular, the pencil $\sigma_{\gamma[0, \infty)}\left[A_{1}, A_{2}\right]$ is non-singular if and only if the product $A_{1}^{-1} A_{2}$ has no negative eigenvalues.

The next lemma describes a simple necessary condition, expressed in terms of matrix pencils, for a strong CQLF to exist for two stable LTI systems. This result concerns general $n$-dimensional systems and is well known in the literature. For instance, see Cohen and Lewkowicz (1997). 
Lemma 2.1 If the stable LTI systems $\Sigma_{A_{1}}, \Sigma_{A_{2}}$ have a strong CQLF, then both of the matrix pencils $\sigma_{\gamma[0, \infty)}\left[A_{1}, A_{2}\right]$ and $\sigma_{\gamma[0, \infty)}\left[A_{1}^{-1}, A_{2}\right]$ are non-singular. Equivalently, the matrix products $A_{1}^{-1} A_{2}, A_{1} A_{2}$ have no negative eigenvalues.

It is important to note that the existence of a strong CQLF for a family of LTI systems is invariant under a change of basis transformation. This is recorded in the following straightforward and well-known lemma, the proof of which involves verifying that if $T \in \mathbb{R}^{n \times n}$ is non-singular, then $A^{T} P+P A<0$ if and only if $\left(T^{-1} A T\right)^{T}\left(T^{T} P T\right)+$ $\left(T^{T} P T\right)\left(T^{-1} A T\right)<0$.

Lemma 2.2 Let $\Sigma_{A_{1}}, \Sigma_{A_{2}}, \ldots, \Sigma_{A_{M}}$ be a family of stable LTI systems and let $T$ be a non-singular matrix in $\mathbb{R}^{n \times n}$. For $i \in\{1, \ldots M\}$, define $\tilde{A}_{i}=T^{-1} A_{i} T$. Then the systems $\Sigma_{A_{1}}, \Sigma_{A_{2}}, \ldots, \Sigma_{A_{M}}$ have a strong CQLF if and only if the systems $\Sigma_{\tilde{A}_{1}}$, $\Sigma_{\tilde{A}_{2}}, \ldots, \Sigma_{A_{M}}$ have a strong $C Q L F$

The next two lemmas are concerned with pairs of systems whose system matrices differ by rank 1. First of all we note that for such systems, one of the two pencils in Lemma 2.1 can never be singular. For a proof of this result consult Laffey et al. (2002). The proof of Lemma 2.4 is contained in the appendices.

Lemma 2.3 Let $A, A+B \in \mathbb{R}^{n \times n}$ be Hurwitz with $\operatorname{rank}(B)=1$. Then the matrix product $A^{-1}(A+B)$ has no negative eigenvalues. Equivalently, the matrix pencil $\sigma_{\gamma[0, \infty)}[A, A+B]$ is non-singular.

Lemma 2.4 Let $A, B \in \mathbb{R}^{n \times n}$ with $A$ Hurwitz and $\operatorname{rank}(B)=1$. Suppose that for some $\lambda_{0}>0$, the matrix product $A\left(A+\lambda_{0} B\right)$ has a negative eigenvalue (the pencil $\sigma_{\gamma[0, \infty)}\left[A^{-1}, A+\lambda_{0} B\right]$ is singular $)$. Then for all $\lambda>\lambda_{0}$, the product $A(A+\lambda B)$ has a negative eigenvalue (the pencil $\sigma_{\gamma[0, \infty)}\left[A^{-1}, A+\lambda B\right]$ is singular).

The following theorem, first proven in Shorten et al. (2003), considers pairs of stable LTI systems for which no strong CQLF exists, but for which a weak CQLF exists with $Q_{i}, i \in\{1,2\}$, of rank $n-1$ and establishes a set of easily verifiable algebraic conditions, that are satisfied when such a weak CQLF exists. It will be later shown that these conditions are found to play an important role in the question of the existence of strong CQLF's for general LTI systems.

Theorem 2.1 Shorten et al. (2003) Let $A_{1}, A_{2}$ be two Hurwitz matrices in $\mathbb{R}^{n \times n}$ such that a solution $P=P^{T} \geq 0$ exists to the non-strict Lyapunov Equations

$$
A_{i}^{T} P+P A_{i}=-Q_{i} \leq 0, i \in\{1,2\}
$$


for some positive semi-definite matrices $Q_{1}, Q_{2}$ both of rank $n-1$. Furthermore suppose that no strong CQLF exists for $\Sigma_{A_{1}}$ and $\Sigma_{A_{2}}$. Under these conditions, at least one of the pencils $\sigma_{\gamma[0, \infty)}\left[A_{1}, A_{2}\right], \sigma_{\gamma[0, \infty)}\left[A_{1}, A_{2}^{-1}\right]$ is singular. Equivalently, at least one of the matrix products $A_{1} A_{2}$ and $A_{1} A_{2}^{-1}$ has a real negative eigenvalue.

The main aim of the rest of the paper is to show how this result provides a unifying framework for two well-known quadratic stability criteria.

Corollary 2.1 Let $A_{1}, A_{2}$ be two Hurwitz matrices in $\mathbb{R}^{n \times n}$. A necessary condition for the existence of a strong $C Q L F$ is that the matrix products $A_{1} A(k)$ and $A_{1} A(k)^{-1}$ have no real negative eigenvalues for all $k \in[0,1]$ where $A(k)=A_{1}+k\left(A_{2}-A_{1}\right)$.

\section{Some new perspectives on old results}

The CQLF existence problem for a finite number of LTI systems is recognised as an analytical problem of extreme difficulty. Although this problem can be solved efficiently numerically using linear matrix inequalities, Boyd and Yang (1989), closedform necessary and sufficient conditions for the existence of a CQLF are currently only known for a few special cases of system classes; in particular, for the case of pairs of second order LTI systems, Shorten and Narendra (2002), and for pairs of $n$-dimensional systems whose systems matrices differ by a rank-1 matrix. In this section we show that both of these cases is a special case of Theorem 2.1. Further, this analysis leads to a new formulation of the SISO circle criterion.

\subsection{Second order systems}

In this section we illustrate the use of Theorem 2.1. We let $\Sigma_{A_{1}}$ and $\Sigma_{A_{2}}$ be stable LTI systems with $A_{1}, A_{2} \in \mathbb{R}^{2 \times 2}$. The following facts follow trivially for second order systems.

(a) If a strong CQLF exists for $\Sigma_{A_{1}}$ and $\Sigma_{A_{2}}$ then the pencils $\sigma_{\gamma[0, \infty)}\left[A_{1}, A_{2}\right]$ and $\sigma_{\gamma[0, \infty)}\left[A_{1}, A_{2}^{-1}\right]$ are necessarily Hurwitz.

(b) If $A_{1}$ and $A_{2}$ satisfy the non-strict Lyapunov equations (3) then the matrices $Q_{1}$ and $Q_{2}$ are both rank 1 (rank $\left.n-1\right)$.

(c) If a strong CQLF does not exist for $\Sigma_{A_{1}}$ and $\Sigma_{A_{2}}$ then a positive constant $d$ exists such that a strong CQLF exists for $\Sigma_{A_{1}-d I}$ and $\Sigma_{A_{2}}$. By continuity a non-negative $d_{1}<d$ exists such that $A_{1}-d_{1} I$ and $A_{2}$ satisfy Theorem 2.1 and one of the pencils $\sigma_{\gamma[0, \infty)}\left[A_{1}-d_{1} I, A_{2}\right]$ and $\sigma_{\gamma[0, \infty)}\left[A_{1}-d_{1} I, A_{2}^{-1}\right]$ is necessarily singular. Hence, it follows that one of the pencils $\sigma_{\gamma[0, \infty)}\left[A_{1}, A_{2}\right]$ and $\sigma_{\gamma[0, \infty)}\left[A_{1}, A_{2}^{-1}\right]$ is not Hurwitz. 
Items (a)-(c) establish the following facts. Given two stable second order LTI systems $\Sigma_{A_{1}}$ and $\Sigma_{A_{2}}$, a necessary condition for the existence of a strong CQLF is that the pencils $\sigma_{\gamma[0, \infty)}\left[A_{1}, A_{2}\right]$ and $\sigma_{\gamma[0, \infty)}\left[A_{1}, A_{2}^{-1}\right]$ are Hurwitz. Conversely, a necessary condition for the non-existence of a strong CQLF is that one of the pencils $\sigma_{\gamma[0, \infty)}\left[A_{1}, A_{2}\right]$ and $\sigma_{\gamma[0, \infty)}\left[A_{1}, A_{2}^{-1}\right]$ is not Hurwitz. Together with Theorem 2.1 these conditions yield the following known result, see Shorten and Narendra (2002), Cohen and Lewkowicz (1997):

$A$ necessary and sufficient condition for the LTI systems $\Sigma_{A_{1}}$ and $\Sigma_{A_{2}}, A_{1}, A_{2} \in$ $\mathbb{R}^{2 \times 2}$, to have a strong $C Q L F$ is that the pencils $\sigma_{\gamma[0, \infty)}\left[A_{1}, A_{2}\right]$ and $\sigma_{\gamma[0, \infty)}\left[A_{1}, A_{2}^{-1}\right]$ are Hurwitz.

\subsection{General systems: The SISO Circle Criterion}

The result presented in Theorem 3.1 below is concerned with the problem of determining necessary and sufficient conditions for a strong CQLF to exist for two LTI systems $\Sigma_{A}, \Sigma_{A-g k^{T}}$ with $A$ and $A-g k^{T}$ in companion form. The result of this theorem can be thought of as a time-domain formulation of the circle criterion. Our main goal in this section is to indicate how Theorem 2.1 above can provide a general setting in which to approach the problem of strong CQLF existence for pairs of LTI systems. With this in mind, the proof we present below illustrates the general nature of the conditions described in Theorem 2.1 by demonstrating that known results for second order systems and those systems covered by the circle criterion can be treated within the framework of the Theorem.

Before stating Theorem 3.1 we make the following preliminary comments.

\section{Preliminaries to Theorem 3.1:}

(i) Because both $A$ and $A-g k^{T}$ are in companion form, we may write;

$$
A=\left(\begin{array}{lllll}
0 & 1 & 0 & \ldots & 0 \\
0 & 0 & 1 & \ldots & 0 \\
\vdots & & & & \\
0 & 0 & 0 & \ldots & 1 \\
-a_{0} & -a_{1} & -a_{2} & \ldots & -a_{n-1}
\end{array}\right), g=\left(\begin{array}{c}
0 \\
0 \\
\vdots \\
0 \\
1
\end{array}\right), k=\left(\begin{array}{c}
k_{0} \\
k_{1} \\
\vdots \\
k_{n-2} \\
k_{n-1}
\end{array}\right)
$$

(ii) If we define the rational function $\Gamma(\omega)$ by

$$
\Gamma(\omega)=1+\operatorname{Re}\left\{k^{T}(j \omega I-A)^{-1} g\right\}
$$

then it follows from the circle criterion that there is a strong CQLF for $\Sigma_{A}$, $\Sigma_{A-g k^{T}}$ if and only if $\Gamma(\omega)>0$ for all real $\omega$ (Kalman (1963), Narendra and 
Goldwyn (1964), Willems (1973)). Furthermore, if we define

$$
\lambda_{c}=\sup \left\{\lambda>0: A \text { and } A-\lambda g k^{T} \text { have a strong CQLF }\right\},
$$

then (provided $\left.\lambda_{c}<\infty\right)$ for $\Gamma_{c}(\omega)=1+\operatorname{Re}\left\{\lambda_{c} k^{T}(j \omega I-A)^{-1} g\right\}$, we have that $\Gamma_{c}(\omega) \geq 0$ for all real $\omega$ and $\Gamma_{c}\left(\omega_{0}\right)=0$ for some $\omega_{0}$.

(iii) We shall need to know how the coefficients of the numerator of $\Gamma$ are related to the entries of $A$ and $k$. As pointed out in Kalman (1963), for any vector $k$ in $\mathbb{R}^{n}$;

$$
k^{T}(s I-A)^{-1} g=\frac{k_{0}+k_{1} s+\ldots+k_{n-1} s^{n-1}}{\operatorname{det}(s I-A)}
$$

and from this it follows that we can write

$$
\Gamma(\omega)=\frac{p_{1}(\omega)}{\operatorname{det}\left(\omega^{2} I+A^{2}\right)}
$$

where $p_{1}$ is a polynomial in $\omega$ of degree $2 n$. Furthermore, as $\operatorname{det}(s I-A)=$ $a_{0}+a_{1} s+\ldots+a_{n-1} s^{n-1}+s^{n}$, we can write

$$
\begin{aligned}
& p_{1}(\omega)=\left[\operatorname{det}\left(\omega^{2} I+A^{2}\right)\right]+\left[k_{0} a_{0}+\left(-k_{0} a_{2}+k_{1} a_{1}-k_{2} a_{0}\right) \omega^{2}\right. \\
+\quad & \left.\left(k_{0} a_{4}-k_{1} a_{3}+k_{2} a_{2}-k_{3} a_{1}+k_{4} a_{0}\right)+\ldots+\left(-k_{n-2}+k_{n-1} a_{n-1}\right) w^{2 n-2}\right] .(6)
\end{aligned}
$$

Note that only even powers of $\omega$ appear in $p_{1}(\omega)$ so that we can also consider $p_{1}$ to be a polynomial in $\omega^{2}$. Now, it follows from (6) that for a given $A \in \mathbb{R}^{n}$ in companion form, the relationship between the entries of the vector $k$ and the coefficients of $p_{1}$ (considered as a polynomial in $\omega^{2}$ ) is described by the affine mapping (from $\mathbb{R}^{n}$ to $\mathbb{R}^{n}$ )

$$
T(k)=\Theta(A)+L(A) k
$$

where $\Theta$ is a vector that depends on the entries of $A$ and $L(A)$ is the linear map given by the matrix (in $\mathbb{R}^{n \times n}$ )

$$
L(A)=\left(\begin{array}{cccccccc}
a_{0} & 0 & 0 & 0 & 0 & \ldots & 0 & 0 \\
-a_{2} & a_{1} & -a_{0} & 0 & 0 & \ldots & 0 & 0 \\
a_{4} & -a_{3} & a_{2} & -a_{1} & a_{0} & \ldots & 0 & 0 \\
\vdots & \vdots & & & & \vdots & \vdots & \\
0 & 0 & 0 & 0 & 0 & \ldots & -1 & a_{n-1}
\end{array}\right)
$$

(iv) Now note that the determinant of $L(A)$ is not independent of the entries of $A$, and hence is not uniformly zero (for instance, the product term $a_{0} a_{1} a_{2} \ldots a_{n-1}$ can only appear once in the expression for the determinant). Thus, for any companion matrix $A$ such that $L(A)$ is singular, it is possible to find another matrix $A^{\prime}$, also in companion form, arbitrarily close to $A$ with $L\left(A^{\prime}\right)$ invertible by perturbing the entries $a_{0}, a_{1}, \ldots, a_{n-1}$. 
Theorem 3.1 Let $A, A-g k^{T}$ be two Hurwitz matrices in companion form in $\mathbb{R}^{n \times n}$ where $g, k$ are column vectors in $\mathbb{R}^{n}$. Assume that the matrix $L(A)$ defined by (8) is non-singular. Then a necessary and sufficient condition for a strong CQLF to exist for the systems $\Sigma_{A}, \Sigma_{A-g k^{T}}$ is that the matrix product $A\left(A-g k^{T}\right)$ has no negative eigenvalues or equivalently, that the matrix pencil $\sigma_{\gamma[0, \infty)}\left[A^{-1}, A-g k^{T}\right]$ is non-singular.

\section{Comments on Theorem 3.1:}

(i) It is important to point out that the assumption that $L(A)$ is invertible is not a very strong restriction on $A$. In fact if we identify the companion matrix

$$
A=\left(\begin{array}{llrlr}
0 & 1 & 0 & \ldots & 0 \\
0 & 0 & 1 & \ldots & 0 \\
\vdots & & & & \\
0 & 0 & 0 & \ldots & 1 \\
-a_{0} & -a_{1} & -a_{2} & \ldots & -a_{n-1}
\end{array}\right)
$$

with the vector $\left(a_{0}, a_{1}, \ldots, a_{n-1}\right)^{T}$ in $\mathbb{R}^{n}$, then the set of those companion matrices for which $L(A)$ is singular would have Lebesgue measure zero.

(ii) Moreover from a practical point of view, if you have two systems in companion form, it is a simple matter to check if $L(A)$ is invertible or not, and if not to adjust the parameters of $A$ to make $L(A)$ invertible.

(iii) Theorem 3.1 can also be extended to the case where the matrices $A$ and $A-g k^{T}$ are not assumed to be in companion form by following Meyer's proof of the extended Kalman Yakubovich Popov lemma given in Meyer (1966) - corresponding to the general case of systems differing by a rank one perturbation.

The crucial point in the proof of Theorem 3.1 is provided by the following lemma which also indicates the relevance of Theorem 2.1 in this context. In the lemma we consider the situation where two systems have just ceased to have a CQLF and we show that under these circumstances there are two systems arbitrarily close to the original systems that satisfy the conditions of Theorem 2.1.

Lemma 3.1 Let $A, A-g k^{T} \in \mathbb{R}^{n \times n}$ be Hurwitz matrices in companion form with $L(A)$ invertible. Suppose that there is no strong $C Q L F$ for $\Sigma_{A}$ and $\Sigma_{A-g k^{T}}$. Furthermore suppose that there is a strong CQLF for $\Sigma_{A}$ and $\Sigma_{A-\lambda g k^{T}}$ for all $\lambda$ with $0<\lambda<1$. Then given any $\epsilon>0$, there is some $k^{\prime} \in \mathbb{R}^{n}$ with $\left\|k-k^{\prime}\right\|<\epsilon$ for which there exists a matrix $P=P^{T} \geq 0$ satisfying

$$
\begin{array}{rlrl}
A^{T} P+P A & =Q_{1} \leq 0 & & \operatorname{rank}\left(Q_{1}\right)=n-1 \\
\left(A-g k^{T}\right)^{T} P+P\left(A-g k^{T}\right) & =Q_{2} \leq 0 & \operatorname{rank}\left(Q_{2}\right)=n-1 .
\end{array}
$$


The proof of Lemma 3.1 is quite long and involved so it is included in the appendix.

Comment: It follows from Theorem 2.1 and Lemma 2.3 that each of the matrix products $A\left(A-g k^{\prime T}\right)$ occurring in the above lemma has a negative real eigenvalue. This in turn implies by the continuous dependence of the eigenvalues of a matrix upon its entries that the matrix product $A\left(A-g k^{T}\right)$ has a negative real eigenvalue.

\section{Proof of Theorem 3.1:}

If there is a strong CQLF for the systems $\Sigma_{A}, \Sigma_{A-g k^{T}}$ given by $V(x)=x^{T} P x$, then it follows from Lemma 2.1 that the product $A\left(A-g k^{T}\right)$ has no negative eigenvalue.

Conversely, suppose there is no strong CQLF for $\Sigma_{A}, \Sigma_{A-g k^{T}}$. Then it follows from the continuous dependence of the eigenvalues of a matrix on the entries of the matrix that for small enough values of $\lambda>0$, the systems $\Sigma_{A}, \Sigma_{A-\lambda g k^{T}}$ will have a strong CQLF. Define $\lambda_{c}=\sup \left\{\lambda>0: \Sigma_{A}\right.$ and $\Sigma_{A-\lambda g k^{T}}$ have a strong CQLF $\}$. Then $\lambda_{c}<1$ and $\Sigma_{A}$ and $\Sigma_{A-\lambda_{c} g k^{T}}$ satisfy the conditions of Lemma 3.1. Thus it follows from the comment above that the matrix product $A\left(A-\lambda_{c} g k^{T}\right)$ has a negative real eigenvalue. It now follows immediately from Lemma 2.4 that the matrix product $A\left(A-g k^{T}\right)$ has a negative real eigenvalue. Q.E.D

\section{General Case and the KYP Lemma in the time domain}

In view of the preceding results and their connection with the SISO circle criterion, it is natural to ask whether or not the generalized Kalman-Yakubovich-Popov (KYP) lemma proven in Meyer (1966) admits a similar time-domain formulation. We provide the answer to this question in this section.

Meyer's result established that for two stable LTI systems $\Sigma_{A}, \Sigma_{A-b c^{T}} A \in \mathbb{R}^{n \times n}$, $b, c \in \mathbb{R}^{n}$, a sufficient condition for the existence of a CQLF is given by

$$
1+\operatorname{Re}\left\{c^{T}(j \omega I-A)^{-1} b\right\}>0 \text { for all } \omega \in \mathbb{R} .
$$

Note that no assumption about $A$ and $A-b c^{T}$ being in companion form is made here (hence the change in notation to avoid confusion). We shall now show that the condition (9) is also necessary for the existence of a CQLF; in fact the matrix product condition described in Theorem 3.1 is equivalent to (9). This extends the work presented in Shorten and Narendra (2003) where an equivalent time-domain formulation of the SISO circle criterion was given.

The following standard lemma is needed for the proof of Theorem 4.1. For details consult Kailath (1980). 
Lemma 4.1 Let $A \in \mathbb{R}^{n \times n}, b, c \in \mathbb{R}^{n}$. Then

$$
\operatorname{det}\left(c^{T}(s I-A)^{-1} b\right)=\frac{\operatorname{det}\left(s I-\left(A-b c^{T}\right)\right)-\operatorname{det}(s I-A)}{\operatorname{det}(s I-A)}
$$

for all complex $s$.

Theorem 4.1 Let $A \in \mathbb{R}^{n \times n}, b, c \in \mathbb{R}^{n}$ be such that $A$ and $A-b c^{T}$ are Hurwitz matrices. Suppose that $A\left(A-b c^{T}\right)$ has no negative eigenvalues. Then the condition (9) holds.

Proof: Suppose that $A\left(A-b c^{T}\right)$ has no negative eigenvalues. Then as $A$ and $A-b c^{T}$ are both Hurwitz their determinants will have the same sign, so it follows that for all $\lambda>0$

$$
\operatorname{det}\left(\lambda I-\left(A-b c^{T}\right) A\right)=\operatorname{det}\left(\lambda I-A^{2}-b c^{T} A\right)>0
$$

Without loss of generality, we may assume that $b c^{T}$ is in one the Jordan canonical forms

$$
\text { (i) } B=\left(\begin{array}{cccc}
\lambda & 0 & \ldots & 0 \\
0 & \ldots & \ldots & 0 \\
\vdots & & & \\
0 & \ldots & \ldots & 0
\end{array}\right) \text {, (ii) } B=\left(\begin{array}{cccc}
0 & \ldots & \ldots & 0 \\
1 & \ldots & \ldots & 0 \\
\vdots & & & \\
0 & \ldots & \ldots & 0
\end{array}\right) \text {. }
$$

If $b c^{T}$ is in either of the above forms then it follows that

$$
\operatorname{det}\left(\lambda I-A^{2}-b c^{T} A\right)=\operatorname{Re}\left\{\operatorname{det}\left(\lambda I-A^{2}-b c^{T} A-\sqrt{\lambda} j b c^{T}\right)\right\},
$$

so writing $\lambda=\omega^{2}$ we have that for all real $\omega$

$$
\operatorname{Re}\left\{\operatorname{det}\left(\omega^{2} I-A^{2}-b c^{T} A-j \omega b c^{T}\right)\right\}>0 .
$$

However a short calculation reveals that

$$
\begin{aligned}
& \operatorname{Re}\left\{\operatorname{det}\left(\omega^{2} I-A^{2}-b c^{T} A-j \omega b c^{T}\right)\right\}>0 \\
& \operatorname{Re}\left\{\operatorname{det}\left(\omega^{2} I+A^{2}\right)+\operatorname{det}\left(\omega^{2} I-A^{2}-b c^{T} A-j \omega b c^{T}\right)-\operatorname{det}\left(\omega^{2} I+A^{2}\right)\right\}>0 \\
& \frac{\operatorname{Re}\left\{\operatorname{det}\left(\omega^{2} I+A^{2}\right)+\operatorname{det}\left(\omega^{2} I-A^{2}-b c^{T} A-j \omega b c^{T}\right)-\operatorname{det}\left(\omega^{2} I+A^{2}\right)\right\}}{\operatorname{det}\left(\omega^{2} I+A^{2}\right)}>0 \\
& \Longleftrightarrow \quad 1+\frac{\operatorname{Re}\left\{\operatorname{det}\left(\omega^{2} I-A^{2}-b c^{T} A-j \omega b c^{T}\right)-\operatorname{det}\left(\omega^{2} I+A^{2}\right)\right\}}{\operatorname{det}\left(\omega^{2} I+A^{2}\right)}>0
\end{aligned}
$$

It follows from (12) that for all $\omega \in \mathbb{R}$

$$
1+\operatorname{Re}\left\{\frac{\operatorname{det}\left(j \omega I-\left(A-b c^{T}\right)\right)-\operatorname{det}(j \omega I-A)}{\operatorname{det}(j \omega I-A)}\right\}>0
$$


and hence from Lemma 4.1 that for all real $\omega$

$$
1+\operatorname{Re}\left\{c^{T}(j \omega I-A)^{-1} b\right\}>0
$$

as claimed. Q.E.D.

\section{Comments on Theorem 4.1}

(i) The above result establishes that condition (9) is necessary as well as sufficient for the existence of a CQLF for $\Sigma_{A}, \Sigma_{A-b c^{T}}$. To the best of the authors' knowledge, this is a new result. To see this, suppose $\Sigma_{A}, \Sigma_{A-b c^{T}}$ have a strong CQLF. Then it follows from Lemma 2.1 that the product $A\left(A-b c^{T}\right)$ has no negative eigenvalues and hence by Theorem 4.1 condition (9) must hold. Note that this also establishes that $A\left(A-b c^{T}\right)$ having no negative eigenvalues is an equivalent time-domain formulation of the condition (9).

(ii) If the matrix product $A\left(A-b c^{T}\right)$ has no negative eigenvalues then from the above theorem and the original result of Meyer (1966), it follows that $\Sigma_{A}$ and $\Sigma_{A-b c^{T}}$ have a strong CQLF. This together with Lemma 2.1 gives the following necessary and sufficient condition for a CQLF to exist for two stable LTI systems differing by rank one.

Theorem 4.2 Let $A, B \in \mathbb{R}^{n \times n}$ with $\operatorname{rank}(B)=1$ and $A$ Hurwitz. Then there is a strong CQLF for the stable LTI systems $\Sigma_{A}$ and $\Sigma_{A-b c^{T}}$ if and only if the matrix product $A\left(A-b c^{T}\right)$ has no negative eigenvalues.

\section{Conclusions}

In this paper, we have presented a result on common quadratic Lyapunov functions, namely Theorem 2.1, and demonstrated that a number of well-known CQLF existence criteria fall within the framework of this result. Considerable empirical evidence indicates to the authors that other system classes will admit treatment within this same framework, and that necessary and sufficient conditions for CQLF existence for these classes may be obtained using Theorem 2.1. The determination of such system classes is currently the subject of ongoing research by the authors and any results obtained in this direction will be reported in future publications.

\section{Acknowledgements}

This work was partially supported by the European Union funded research training network Multi-Agent Control, HPRN-CT-1999-00107 ${ }^{1}$ and by the Enterprise Ireland

\footnotetext{
${ }^{1}$ This work is the sole responsibility of the authors and does not reflect the European Union's opinion
} 
grant SC/2000/084/Y. Neither the European Union or Enterprise Ireland is responsible for any use of data appearing in this publication.

\section{References}

Boyd, S. and Yang, Q. (1989). Structured and simultaneous Lyapunov functions for system stability problems. Internat. J. Control, 49(6), 2215-2240.

Cohen, N. and Lewkowicz, N. (1997). Convex invertible cones and the Lyapunov equation. Lin. Alg. and its Appl., 250(1), 105-131.

Kailath, T. (1980). Linear Systems. Prentice Hall, New Jersey.

Kalman, R. (1963). Lyapunov functions for the problem of Lur'e in automatic control. Proc. Natl. Acad. Sci., 49(2), 201-205.

Laffey, T., Shorten, R., and O'Cairbre, F. (2002). On the stability of convex sums of rank-1 perturbed matrices. In Proceedings of the American Control Conference.

Meyer, K. R. (1966). On the existence of Lyapunov functions for the problem of Lur'e. SIAM Journal of Control, 3(3), 373-383.

Narendra, K. and Goldwyn, R. (1964). A geometrical criterion for the stability of certain non-linear non-autonomous systems. IEEE Transactions on Circuit Theory, 11(3), 406-407.

Shorten, R. N. and Narendra, K. (2002). Necessary and sufficient conditions for the existence of a common quadratic Lyapunov function for a finite number of stable second order linear time-invariant systems. International Journal of Adaptive Control and Signal Processing, 16, 709-728.

Shorten, R. N. and Narendra, K. S. (2003). A note on common quadratic Lyapunov functions. IEEE Transactions on Automatic Control, 48(4), 618-621.

Shorten, R. N., Narendra, K. S., and Mason, O. (2003). A result on common quadratic Lyapunov functions. IEEE Transactions on Automatic Control, 48(1), 110-113.

Willems, J. (1973). The circle criterion and quadratic Lyapunov functions for stability analysis. IEEE Transactions on Automatic Control, 18, 184. 


\section{Appendix}

\section{Proof of Lemma 2.1:}

If there is a strong CQLF for the systems $\Sigma_{A_{1}}, \Sigma_{A_{2}}$ given by $V(x)=x^{T} P x$, then $V(x)$ will also be a strong quadratic Lyapunov function for each of the systems $\Sigma_{\sigma_{\gamma[0, \infty)}\left[A_{1}, A_{2}\right]}$, $\Sigma_{\sigma_{\gamma[0, \infty)}\left[A_{1}^{-1}, A_{2}\right]}$. Thus each of the matrices in the pencils $\sigma_{\gamma[0, \infty)}\left[A_{1}, A_{2}\right], \sigma_{\gamma[0, \infty)}\left[A_{1}^{-1}, A_{2}\right]$ is Hurwitz and hence non-singular, and the matrix products $A_{1}^{-1} A_{2}, A_{1} A_{2}$ have no negative eigenvalues.

\section{Proof of Lemma 2.4:}

Without loss of generality, we may assume that $B$ is in one the Jordan canonical forms given by (10). Suppose that $\lambda_{0}>0$ is such that $A\left(A+\lambda_{0} B\right)$ has a real negative eigenvalue. It follows that for this $\lambda_{0}$ there is some $\gamma_{0}>0$ such that $A^{-1}+\gamma_{0}\left(A+\lambda_{0} B\right)$ is singular. Thus

$$
\operatorname{det}\left(A^{-1}+\gamma_{0}\left(A+\lambda_{0} B\right)\right)=\operatorname{det}\left(\left(A^{-1}+\gamma_{0} A\right)+\lambda_{0} \gamma_{0} B\right)=0 .
$$

For any $\gamma>0$, it follows from considering the form of the matrix $\left(A^{-1}+\gamma A\right)+\lambda_{0} \gamma B$, that we may write

$$
\operatorname{det}\left(\left(A^{-1}+\gamma A\right)+\lambda_{0} \gamma B\right)=M(\gamma)+\lambda_{0} N(\gamma)
$$

where $M$ and $N$ are polynomials in $\gamma$.

We now note the following facts about the polynomials $M$ and $N$.

(i) $M(\gamma)=\operatorname{det}\left(A^{-1}+\gamma A\right)$ is non-zero and of the same sign for all $\gamma>0\left(A^{-1}+\gamma A\right)$ is always Hurwitz).

(ii) $M(0)+\lambda N(0)=M(0)$ for any $\lambda>0$.

For convenience, assume that $M(\gamma)>0$ for all $\gamma>0$. Now, $M\left(\gamma_{0}\right)+\lambda_{0} N\left(\gamma_{0}\right)=0$ and as $M\left(\gamma_{0}\right)>0$, we must have $N\left(\gamma_{0}\right)<0$. Then for any $\lambda>\lambda_{0}$

$$
M\left(\gamma_{0}\right)+\lambda N\left(\gamma_{0}\right)<M\left(\gamma_{0}\right)+\lambda_{0} N\left(\gamma_{0}\right)=0
$$

But $M(0)+\lambda N(0)=M(0)>0$, so by the intermediate value theorem, there is some $\gamma_{1}$ with $0<\gamma_{1}<\gamma_{0}$ such that

$$
\operatorname{det}\left(A^{-1}+\lambda\left(A+\gamma_{1} B\right)\right)=M\left(\gamma_{1}\right)+\lambda N\left(\gamma_{1}\right)=0
$$

and hence the matrix product $A(A+\lambda B)$ has a real negative eigenvalue as claimed.

Proof of Lemma 3.1: Under the hypotheses of the lemma, we know that

$$
1+\operatorname{Re}\left\{k^{T}(j \omega I-A)^{-1} g\right\} \geq 0
$$


for all $\omega \in \mathbb{R}$ and that there is definitely some value of $\omega$ for which this expression is zero. The proof of this lemma is broken into a number of steps.

\section{Step 1:}

The first step is to prove that it is possible to find a vector $k^{\prime}$ arbitrarily close to $k$ such that

$$
1+\operatorname{Re}\left\{k^{\prime T}(j \omega I-A)^{-1} g\right\} \geq 0 \text { for all } \omega \in \mathbb{R}
$$

with a single real zero of multiplicity 2 at some $\omega_{c}$. By assumption the mapping $T$ defined in (7) is invertible with continuous inverse $T^{-1}$. Throughout the proof we shall identify a polynomial in $\omega^{2}$ of degree $n-1$ with the vector in $\mathbb{R}^{n}$ defined by its coefficients. Write $p=T(k)$. Then for any $\epsilon>0$ there is some $\delta>0$ such that $\left\|p-p^{\prime}\right\|<\delta$ implies $\left\|k-T^{-1}\left(p^{\prime}\right)\right\|<\epsilon$.

Now $p(\omega) \geq 0$ for all $\omega \in \mathbb{R}$, so the zeroes of $p$ occur as complex conjugate pairs or as real zeroes of even multiplicity. If $p$ has more than a single real zero of multiplicity 2 , then by replacing terms like $\left(\omega-\omega_{1}\right)\left(\omega-\omega_{1}\right)$ in the linear factorization of $p$ by the terms $\left(\omega-\left(\omega_{1}+j \delta_{1}\right)\left(\omega-\left(\omega_{1}-j \delta_{1}\right)\right.\right.$, we can find a polynomial $p^{\prime}$ whose coefficients are arbitrarily close to those of the original $p$ such that

(i) $p^{\prime}(\omega) \geq 0$ for all $\omega \in \mathbb{R}$

(ii) $p^{\prime}$ has a single zero of multiplicity two

(iii) Only the even coefficients of $p^{\prime}$ are non-zero

In particular, we can choose such a $p^{\prime}$ with $\left\|p^{\prime}-p\right\|<\delta$ and thus defining $k^{\prime}=T^{-1}\left(p^{\prime}\right)$, we have $\left\|k^{\prime}-k\right\|<\epsilon$ and

$$
1+R e\left\{k^{T}(j \omega I-A)^{-1} g\right\} \geq 0
$$

with a single real zero of multiplicity 2 as required. We shall show that this $k^{\prime}$ is the vector required in the lemma.

Step 2:

For the rest of the proof, we shall write $S(\omega)$ for the expression $j \omega I-A$. The next stage is to show that for the matrices $A$ and $A-g k^{\prime T}$, it is possible to find a positive semi-definite matrix $R \geq 0$ of rank $n-2$ such that

$$
1+\operatorname{Re}\left\{\left(k^{\prime}\right)^{T} S(\omega)^{-1} g\right\} \geq g^{T}\left(S(\omega)^{*}\right)^{-1} R S(\omega)^{-1} g
$$

To begin with, choose any positive semi-definite matrix $R^{\prime}$ in $\mathbb{R}^{n \times n}$ of rank $n-2$ such that $R^{\prime} S\left(\omega_{c}\right)^{-1} g=0$ where $\omega_{c}$ is the only real zero of $1+2 \operatorname{Re}\left\{\left(k^{\prime}\right)^{T} S(\omega)^{-1} g\right\}$. It is 
possible to scale this $R$ so that it satisfies the condition (15). First of all note that

$$
g^{T}\left(S(\omega)^{*}\right)^{-1} R^{\prime} S(\omega)^{-1} g=\frac{p_{2}(\omega)}{\operatorname{det}\left(\omega^{2} I+A^{2}\right)}
$$

where $p_{2}$ is a polynomial of degree $2 n-2$ in $\omega$. Similarly, write

$$
1+\operatorname{Re}\left\{\left(k^{\prime}\right)^{T} S(\omega)^{-1} g\right\}=\frac{p_{1}(\omega)}{\operatorname{det}\left(\omega^{2} I+A^{2}\right)}
$$

where $p_{1}$ is a polynomial of degree $2 n$ in $\omega$ whose leading term is $\omega^{2 n}$. Now as $|\omega|$ tends to infinity, the expression

$$
1+\operatorname{Re}\left\{\left(k^{\prime}\right)^{T} S(\omega)^{-1} g\right\}-g^{T}\left(S(\omega)^{*}\right)^{-1} R^{\prime} S(\omega)^{-1} g
$$

tends to 1 . Thus, by continuity there is some constant $K>0$ such that $1+2 \operatorname{Re}\left\{\left(k^{\prime}\right)^{T} S(\omega)^{-1} g\right\}-$ $g^{T}\left(S(\omega)^{*}\right)^{-1} R^{\prime} S(\omega)^{-1} g>0$ for all $\omega$ with $|\omega|>K$. Furthermore

$$
\begin{aligned}
1+\operatorname{Re}\left\{\left(k^{\prime}\right)^{T} S(\omega)^{-1} g\right\}-g^{T}\left(S(\omega)^{*}\right)^{-1} R^{\prime} S(\omega)^{-1} g & =\frac{p_{1}(\omega)-p_{2}(\omega)}{\operatorname{det}\left(\omega^{2} I+A^{2}\right)} \\
& =\left(\omega-\omega_{c}\right)^{2} \frac{\left(p_{1}^{\prime}(\omega)-p_{2}^{\prime}(\omega)\right)}{\operatorname{det}\left(\omega^{2} I+A^{2}\right)}
\end{aligned}
$$

with $p_{1}^{\prime}(\omega)>0$ for all $\omega$. Thus there is some constant $M_{1}>0$ such that $p_{1}^{\prime}(\omega) \geq M_{1}$ for $\omega$ in the compact interval $[-K, K]$. Furthermore there is some $M_{2}>0$ such that $p_{2}^{\prime}(\omega) \leq M_{2}$ for all $\omega \in[-K, K]$. If we now choose some constant $C>0$ such that $C<\min \left\{M_{1} / M_{2}, 1\right\}$, then by separately considering the cases of $\omega \in[-K, K]$ and $|\omega|>K$ we see that

$$
1+\operatorname{Re}\left\{k^{\prime T} S(\omega)^{-1} b\right\} \geq g^{T}\left(S(\omega)^{*}\right)^{-1}\left(C R^{\prime}\right) S(\omega)^{-1} g
$$

for all $\omega \in \mathbb{R}$. So $R=C R^{\prime}$ is a positive semi-definite matrix of rank $n-2$ satisfying condition (15) as claimed.

Now the numerator of the rational function

$$
1+\operatorname{Re}\left\{k^{\prime T} S(\omega)^{-1} b\right\}-g^{T}\left(S(\omega)^{*}\right)^{-1}(R) S(\omega)^{-1} g
$$

has real coefficients, is non-negative and can be thought of as a polynomial of degree $n$ in $-\omega^{2}$. By arguments identical to those presented in Kalman (1963), it follows that there is some polynomial $\theta$ of degree $n$ with real coefficients and leading coefficient equal to one such that

$$
1+\operatorname{Re}\left\{k^{\prime T} S(\omega)^{-1} b\right\}-g^{T}\left(S(\omega)^{*}\right)^{-1}(R) S(\omega)^{-1} g=\frac{|\theta(j \omega)|^{2}}{\operatorname{det}\left(\omega^{2} I+A^{2}\right)} .
$$


As the leading coefficient of $\theta$ is one, the polynomial $-\theta(s)+\operatorname{det}(s I-A)$ has degree $n-1$, and thus by (5), there is some real vector $q$ (the vector formed with the coefficients of $-\theta(s)+\operatorname{det}(s I-A))$ such that

$$
\frac{-\theta(j \omega)}{\operatorname{det}(S(\omega))}+1=q^{T} S(\omega)^{-1} g
$$

and hence

$$
\begin{aligned}
\frac{|\theta(j \omega)|^{2}}{\operatorname{det}\left(\omega^{2} I+A^{2}\right)} & =1+\operatorname{Re}\left\{k^{\prime T} S(\omega)^{-1} g\right\}-g^{T}\left(S(\omega)^{*}\right)^{-1}(R) S(\omega)^{-1} g \\
& =\left|q^{T} S(\omega)^{-1} g-1\right|^{2}
\end{aligned}
$$

Step 3:

We now show that (16) means that there is a positive semi-definite matrix $P$ such that

$$
\begin{aligned}
A^{T} P+P A & =-q q^{T}-R \\
P g & =q+k^{\prime}
\end{aligned}
$$

As $A$ is Hurwitz, we can certainly find a positive semi-definite matrix $P$ such that $A^{T} P+P A=-q q^{T}-R$. This $P$ then satisfies

$$
S(\omega)^{*} P+P S(\omega)=q q^{T}+R
$$

Next expand the identity (16) to get

$$
\begin{aligned}
1 & +\operatorname{Re}\left\{k^{T} S(\omega)^{-1} g\right\}-g^{T}\left(S(\omega)^{*}\right)^{-1}(R) S(\omega)^{-1} g \\
& =\left(g^{T}\left(S(\omega)^{*}\right)^{-1} q-1\right)\left(q^{T} S(\omega)^{-1} g-1\right) \\
& =1-2 \operatorname{Re}\left\{q^{T} S(\omega)^{-1} g\right\}+g^{T}\left(S(\omega)^{*}\right)^{-1} q q^{T} S(\omega)^{-1} g
\end{aligned}
$$

Collecting the terms in (19) and using (18) to substitute for $q q^{T}+R$ we find that

$$
\operatorname{Re}\left\{\left(k^{\prime} / 2+q-P g\right)^{T} S^{-1} g\right\}=0 \text { for all } \omega \in \mathbb{R} .
$$

But this implies that $k^{\prime} / 2+q-P g=0$ or $P g-k^{\prime} / 2=q$. (This follows from the fact that the matrix $L(A)$ in (8) is assumed to be invertible.)

Step 4:

The next step is to show that the matrix $P$ in (17) is a weak CQLF for $A, A-g k^{\prime T}$ with

$$
\begin{aligned}
A^{T} P+P A & =Q_{1} \leq 0 \\
\left(A-g k^{T}\right)^{T} P+P\left(A-g k^{\prime T}\right) & =Q_{2} \leq 0
\end{aligned}
$$


where $Q_{1}$ and $Q_{2}$ are both of rank $n-1$.

Obviously, $A^{T} P+P A=-q q^{T}-R=Q_{1} \leq 0$. We shall show later that the rank of $Q_{1}$ is $n-1$. First of all consider

$$
\begin{aligned}
\left(A-g k^{T}\right)^{T} P+P\left(A-g k^{T}\right) & =-q q^{T}-R-k^{\prime} g^{T} P-P g k^{\prime T} \\
& =-q q^{T}-R-k^{\prime}\left(k^{\prime} / 2+q\right)^{T}-\left(k^{\prime} / 2+q\right) k^{T} \\
& =-R-\left(k^{\prime}+q\right)\left(k^{\prime}+q\right)^{T} \leq 0
\end{aligned}
$$

So $P$ defines a weak CQLF for $A$ and $A-g k^{\prime T}$ as claimed. Next as $R$ is of rank $n-2$, we can write $R=\sum_{i=1}^{n-2} v_{i} v_{i}^{T}$ for $n-2$ linearly independent vectors $v_{1}, \ldots v_{n-2}$. Also recall that $S\left(\omega_{c}\right)^{-1} g$ is a zero eigenvector of $R$. If the rank of $q q^{T}+R$ was less than $n-1$, then $q$ would lie in the span of $v_{1}, \ldots, v_{n-2}$, and thus $q^{T}\left(S \omega_{c}\right)^{-1} g=0$. But it would then follow from (16) that $1=0$ - an absurdity. Thus the rank of $q q^{T}+R$ must be $n-1$. A similar argument shows that $R+\left(k^{\prime}+q\right)\left(k^{\prime}+q\right)^{T}$ is of rank $n-1$. This completes the proof of Lemma 3.1. 